\title{
Remarks on Spectra of Modular Operators of von Neumann Algebras
}

\author{
Huzihiro Araki ${ }^{\star}$ \\ Department of Mathematics, Queen's University, Kingston, Ontario, Canada
}

Received June 20, 1972

\begin{abstract}
It is shown that if $\varrho$ is an invariant state of an asymptotically abelian $C^{*}$ algebra $\mathfrak{A}$, then the spectrum of modular operator for $\varrho$ is contained in the spectrum of any other modular operator for the von Neumann algebra $\pi_{e}(\mathfrak{Q})^{\prime \prime}$.

It is also shown that a modular operator can not have an isolated spectrum with a finite multiplicity at 1 unless the associated Hilbert space is of finite dimension. It is further shown that if a modular operator has an isolated spectrum with a finite multiplicity at $x \neq 1$, then the von Neumann algebra $\mathfrak{R}$ is a direct sum of $\mathfrak{R}_{1}$ and $\mathfrak{R}_{2}$ where $\mathfrak{R}_{1}$ is represented on a finite dimensional Hilbert space and the modular operator for $\mathfrak{R}_{2}$ does not have its spectrum at $x$.

Applications to Connes invariant are given.
\end{abstract}

\section{§1. Preliminaries}

A net of operators $Q_{\alpha}$ in a von Neumann algebra $\Re$ is called weakly (or strongly) central if there exists weakly total self adjoint subset $\mathfrak{R}_{0}$ of $\Re$ such that $\left[Q_{\alpha}, Q\right] \rightarrow 0$ weakly (or strongly) for every $Q \in \mathfrak{R}_{0}$. If $Q_{\alpha}$ is uniformly bounded and weakly central, then $\mathrm{w}-\lim \left[Q_{\alpha}, Q\right]=0$ for all $Q \in \mathfrak{R}([1])$.

A subset $\mathfrak{A}$ of $\mathfrak{R}$ is called weakly (or strongly) $\tau_{\alpha}$ central relative to a net of * automorphisms $\tau_{\alpha}$ of $\mathfrak{R}$ if $\tau_{\alpha} Q$ is weakly (or strongly) central in $\mathfrak{R}$ for each $Q \in \mathfrak{A}$.

For any state $\varrho$ of $\Re$, we denote by $H_{\varrho}, \pi_{\varrho}$ and $\Omega_{\varrho}$ a Hilbert space, a representation of $\Re$ on $H_{\varrho}$ and a cyclic vector in $H_{\varrho}$ associated with $\varrho$ through the relation

$$
\varrho(Q)=\left(\Omega_{\varrho}, \pi_{\varrho}(Q) \Omega_{\varrho}\right), \quad Q \in \mathfrak{R} .
$$

$J_{\varrho}$ and $\Delta_{\varrho}$ denote modular conjugation operator and modular operator for $\Omega_{\varrho}$ when $\varrho$ is faithful. $\bar{\tau}_{\varrho}(t) Q \equiv \Delta_{\varrho}^{i t} Q \Delta_{\varrho}^{-i t}$.

If $\varrho$ is $\tau_{\alpha}$ invariant, then there exists a unitary $U_{\alpha}$ such that $U_{\alpha} \pi_{\varrho}(Q) \Omega_{\varrho}$ $=\pi_{\varrho}\left(\tau_{\alpha} Q\right) \Omega_{\varrho}$ for all $Q \in \mathfrak{R}$. We denote $U_{\alpha} Q U_{\alpha}^{*}=\bar{\tau}_{\alpha} Q$ for $Q \in \mathscr{B}\left(H_{\varrho}\right)$. The following result has been obtained in [1]. (See also appendix.)

* On leave from Research Institute for Mathematical Sciences, Kyoto University, Kyoto, Japan. 
Lemma 1. Let a weakly dense * subalgebra $\mathfrak{A}$ of $\mathfrak{R}$ be strongly $\tau_{\alpha}$ central, $\varrho$ be a faithful normal state on $\mathfrak{R}$, invariant under all $\tau_{\alpha}$ and $\hat{\mathfrak{A}}$ be the $C^{*}$ algebra generated by $\pi_{\varrho}(\mathfrak{Q}) j_{\varrho}\left\{\pi_{\varrho}(\mathfrak{H})\right\}$ where $j_{\varrho}(Q)=J_{\varrho} Q J_{\varrho}$. Let $\hat{\varrho}$ denote the vector state on $\mathscr{B}\left(H_{\varrho}\right)$ by the vector $\Omega_{\varrho}$ and $\varrho^{\prime}$ be any normal state on $\mathscr{B}\left(H_{\varrho}\right)$, such that its restriction to the center $\left.3=\pi_{\varrho}(\Re) \bigcap \pi_{\varrho} \mid \mathfrak{R}\right)^{\prime}$ of $\mathfrak{R}$ is the same as that of $\hat{\varrho}: \hat{\varrho}(z)=\varrho^{\prime}(z)$ for all $z \in \mathcal{3}$. Then

$$
\lim \varrho^{\prime}\left(\bar{\tau}_{\alpha} Q\right)=\hat{\varrho}(Q), \quad Q \in \hat{\mathfrak{A}} .
$$

To achieve the situation $\left.\varrho^{\prime}\right|_{3}=\left.\varrho\right|_{3}$, we use the following commutative Radon-Nikodym Theorem. Here, $s(\varrho)$ denotes the support projection of $\varrho$.

Lemma 2. Let $\varrho_{1}$ and $\varrho_{2}$ be normal states of a commutative von Neumann algebra 3 and $s\left(\varrho_{1}\right) \geqq s\left(\varrho_{2}\right)$. (The last condition is automatically fulfilled if $\varrho_{1}$ is faithful.) Then there exists a non-negative self adjoint operator $A^{3}\left(\varrho_{2} / \varrho_{1}\right)$ affiliated with 3 such that $\Omega_{\varrho_{1}}$ is in the domain of $\pi_{\varrho_{1}}\left(A^{3}\left(\varrho_{2} / \varrho_{1}\right)\right)\left(\equiv \int \lambda \mathrm{d} \pi_{\varrho_{1}}\left(E_{\lambda}\right)\right.$ if $\left.A^{3}\left(\varrho_{2} / \varrho_{1}\right)=\int \lambda \mathrm{d} E_{\lambda}\right)$ and the vector state on 3 by the vector $\Omega^{\prime} \equiv \pi_{\varrho_{1}}\left(A^{3}\left(\varrho_{2} / \varrho_{1}\right)\right) \Omega_{\varrho_{1}}$ is $\varrho_{2}$.

$A\left(\varrho_{2} / \varrho_{1}\right)$ is the positive square root of Radon-Nikodym derivate in measure theoretical sense.

Lemma 3. Let $\Re$ be a von Neumann algebra on $H$ and $\Omega$ and $\Omega^{\prime}$ be two cyclic and separating vectors related by $\Omega^{\prime}=A \Omega$ where $A$ is a positive self adjoint operator affiliated with center $3=\mathfrak{R} \cap \mathfrak{R}^{\prime}$. Then $\Delta_{\Omega}=\Delta_{\Omega^{\prime}}$.

Proof. Let $z \in 3, z=z^{*}$ and $S_{\Omega}=J_{\Omega} \Delta_{\Omega}^{1 / 2}$.

Then

$$
S_{\Omega} Q z \Omega=z Q^{*} \Omega=Q^{*} z \Omega, \quad Q \in \mathfrak{R} .
$$

Let $A=\int \lambda \mathrm{d} E_{\lambda}, A_{L}=A E_{L}$. Then $A_{L} \in 3, A_{L}{ }^{*}=A_{L}$. Further,

$$
\lim _{L \rightarrow+\infty} Q A_{L} \Omega=Q \Omega^{\prime}, \quad \lim _{L \rightarrow+\infty} Q^{*} A_{L} \Omega=Q^{*} \Omega^{\prime}
$$

for $Q \in \mathfrak{R}$. Since $S_{\Omega}$ is closed, we have

$$
S_{\Omega} Q \Omega^{\prime}=Q^{*} \Omega^{\prime}=S_{\Omega^{\prime}} Q \Omega^{\prime} .
$$

Hence $S_{\Omega} \supset S_{\Omega^{\prime}}$. Since $\Omega=A^{-1} \Omega^{\prime}$, we have $S_{\Omega^{\prime}} \supset S_{\Omega}$. Therefore $S_{\Omega}=S_{\Omega^{\prime}}$, which implies $\Delta_{\Omega}=S_{\Omega}{ }^{*} S_{\Omega}=S_{\Omega^{\prime}}{ }^{*} S_{\Omega^{\prime}}=\Delta_{\Omega^{\prime}}$. Q.E.D.

The following Lemma has been given by Connes [2].

Lemma 4. $t \in[0, \infty)$ is in the spectrum of $\Delta_{\Omega}$ if and only if there exist operators $x \in \mathfrak{R}$ and $y \in \mathfrak{R}^{\prime}$ for each given $\varepsilon>0$ such that $\|x \Omega\|=1$, $\left\|t^{1 / 2} x \Omega-y \Omega\right\|<\varepsilon$ and $\left\|x^{*} \Omega-t^{1 / 2} y^{*} \Omega\right\|<\varepsilon$. 


\section{§ 2. Invariant State of Asymptotically Abelian System}

Theorem 1. Let $\tau_{\alpha}$ be a net of * automorphisms of $\Re$ such that a weakly dense sub * algebra $\mathfrak{A}$ of $\mathfrak{R}$ is strongly $\tau_{\alpha}$ central and $\varrho$ be a faithful normal state of $\mathfrak{R}$, invariant under all $\tau_{\alpha}$. Then the spectrum of $\Delta_{\varrho}$ is contained in the spectrum of $\Delta_{\varrho^{\prime}}$ for any faithful normal state $\varrho^{\prime}$ on $\mathfrak{R}$.

Remark. This theorem with an assumption of strong clustering has been given by St $\phi$ rmer [4].

Proof. Let $t \in[0, \infty)$ be in the spectrum of $\Delta_{Q}$ and $\varepsilon>0$ be given. By Lemma 4, there exists $x \in \mathfrak{R}$ and $y \in \mathfrak{R}^{\prime}$ satisfying

$$
\begin{gathered}
\left\|x \Omega_{\varrho}\right\|=1, \\
\left\|t^{1 / 2} x \Omega_{\varrho}-y \Omega_{\varrho}\right\|<\varepsilon / 4, \\
\left\|x^{*} \Omega_{\varrho}-t^{1 / 2} y^{*} \Omega_{\varrho}\right\|<\varepsilon / 4 .
\end{gathered}
$$

Since $\mathfrak{A}$ is a self adjoint linear weakly dense subset of $\mathfrak{R}$, it is * strongly dense in $\mathfrak{R}$. Hence there exist $x_{1} \in \pi_{\varrho}(\mathfrak{a})$ and $y_{1} \in J_{\varrho} \pi_{\varrho}(\mathfrak{a}) J_{\varrho}$ such that

$$
\begin{gathered}
\left\|x_{1} \Omega_{\varrho}\right\|=1, \\
\left\|t^{1 / 2}\left(x-x_{1}\right) \Omega_{\varrho}-\left(y-y_{1}\right) \Omega_{\varrho}\right\|<\varepsilon / 4, \\
\left\|\left(x^{*}-x_{1}^{*}\right) \Omega_{\varrho}-t^{1 / 2}\left(y^{*}-y_{1}^{*}\right) \Omega_{\varrho}\right\|<\varepsilon / 4 .
\end{gathered}
$$

Since $\Omega_{\varrho}$ is cyclic and separating for $\pi_{\varrho}(\mathfrak{R}) \sim \mathfrak{R}$, there exists a vector $\Omega_{\varrho^{\prime}} \in H_{\varrho}$ such that the vector state by $\Omega_{\varrho^{\prime}}$ on $\Re$ is $\varrho^{\prime}$. By Lemma 2 , there exists a positive self adjoint operator $z$ affiliated with $3=\pi_{\varrho}(\mathfrak{R}) \cap \pi_{\varrho}(\mathfrak{R})^{\prime}$ such that $z \Omega_{\varrho^{\prime}} \equiv \Omega^{\prime}$ gives the same vector state on 3 as $\Omega_{\varrho^{\prime}}$. Let $\varrho^{\prime \prime}$ be the vector state on $\mathscr{B}\left(H_{\varrho}\right)$ by the vector $\Omega^{\prime}$.

By Lemma 1, there exists $\alpha$ such that

$$
\begin{gathered}
\left|\left(\varrho^{\prime \prime}-\hat{\varrho}\right)\left(\bar{\tau}_{\alpha}\left(x_{1}{ }^{*} x_{1}\right)\right)\right|<1 / 2, \\
\left|\left(\varrho^{\prime \prime}-\varrho\right)\left(\bar{\tau}_{\alpha}\left\{\left(t^{1 / 2} x_{1}-y_{1}\right)^{*}\left(t^{1 / 2} x_{1}-y_{1}\right)\right\}\right)\right|<\varepsilon^{2} / 4, \\
\left|\left(\varrho^{\prime \prime}-\varrho\right)\left(\bar{\tau}_{\alpha}\left\{\left(x_{1}{ }^{*}-t^{1 / 2} y_{1}{ }^{*}\right)^{*}\left(x_{1}{ }^{*}-t^{1 / 2} y_{1}{ }^{*}\right)\right\}\right)\right|<\varepsilon^{2} / 4 .
\end{gathered}
$$

We define $\lambda=\varrho^{\prime \prime}\left(\bar{\tau}_{\alpha}\left(x_{1}{ }^{*} x_{1}\right)\right)^{1 / 2}$. Then $\lambda^{2}>1-1 / 2=1 / 2$, due to $\hat{\varrho}\left(\bar{\tau}_{\alpha}\left(x_{1}^{*} x_{1}\right)\right)=\hat{\varrho}\left(x_{1} * x_{1}\right)=1$. We define

$$
x_{2}=\lambda^{-1} \bar{\tau}_{\alpha} x_{1}, \quad y_{2}=\lambda^{-1} \bar{\tau}_{\alpha} y_{1} .
$$

By previous estimates and $\bar{\tau}_{\alpha}$ invariance of $\varrho$, we have

$$
\begin{gathered}
\varrho^{\prime \prime}\left(x_{2}{ }^{*} x_{2}\right)=1, \\
\varrho^{\prime \prime}\left(\left(t^{1 / 2} x_{2}-y_{2}\right)^{*}\left(t^{1 / 2} x_{2}-y_{2}\right)\right)<\varepsilon^{2}, \\
\varrho^{\prime \prime}\left(\left(x_{2}{ }^{*}-t^{1 / 2} y_{2}\right)^{*}\left(x_{2}{ }^{*}-t^{1 / 2} y_{2}{ }^{*}\right)\right)<\varepsilon^{2} .
\end{gathered}
$$


Since $x_{2} \in \pi_{\varrho}(\Re), y_{2} \in \pi_{\varrho}(\Re)^{\prime}, t$ is in the spectrum of $\Delta_{\Omega^{\prime}}$ by Lemma 4 . By Lemma 3, $\Delta_{\Omega^{\prime}}=\Delta_{\Omega_{\Omega^{\prime}}}$. Hence $t$ is in the spectrum of $\Delta_{\varrho^{\prime}}$. Q.E.D.

\section{§3. Isolated Spectrum with a Finite Multiplicity at 1}

Theorem 2. If 1 is an isolated spectrum of $\Delta_{\varrho}$ with a multiplicity $n$, then $\operatorname{dim} H_{\varrho} \leqq n^{2}$.

We need a few preparations for the proof of this Theorem. Let $\mathfrak{A}$ be any weakly dense $\bar{\tau}_{\varrho}(t)$ invariant norm closed linear subset of $\pi_{\varrho}(\Re)$. Let $\Delta_{\varrho}=\int_{-\infty}^{\infty} e^{\lambda} \mathrm{d} E_{\lambda}$. For any bounded open interval $I=(a, b)$, define $\mathfrak{A}_{I}$ as the set of all operators $Q$ in $\mathfrak{A}$ such that

$$
\begin{aligned}
& Q H((\alpha, \beta)) \subset H((\alpha+a, \beta+b)), \\
& H((\alpha, \beta))=\left(E_{\beta-0}-E_{\alpha+0}\right) H_{\varrho} .
\end{aligned}
$$

From the definition

$$
\mathfrak{U}_{I_{1}} \mathfrak{A}_{I_{2}} \subset \hat{\mathfrak{R}}_{I_{1}+I_{2}}, \quad \hat{\mathfrak{R}}=\pi_{\varrho}(\mathfrak{R}) .
$$

Let $I \subset C J$ denotes $\bar{I} \subset J$ where $\bar{I}$ is the closure of $I$.

Lemma 5. $H(I)$ is the closure of $\bigcup_{J \subset \subset I} \mathfrak{A}_{J} \Omega_{Q}$.

Proof. Since $\Omega_{\varrho} \in H\left(I^{\prime \prime}\right)$ for any $I^{\prime \prime}$ containing 0 , we have $\mathfrak{A}_{J} \Omega_{\varrho} \subset H(I)$ if $J \subset C I$.

Since $\bigcup_{J \subset \subset I} A_{J} \Omega_{Q}$ is a linear set, it is enough to show that for any unit vector $\Phi \in H(I)$, there exists $J \subset C I$ and $Q \in \mathfrak{A}_{J}$ such that $\left(Q \Omega_{\varrho}, \Phi\right) \neq 0$.

Let $I=(a, b)$. By definition, there exist $a<a^{\prime}<b^{\prime}<b$ such that $\left\|\left(E_{b^{\prime}-0}-E_{a^{\prime}+0}\right) \Phi\right\| \neq 0$. $\left(\|\Phi\|=1\right.$ by assumption.) Let $J=\left(a^{\prime}, b^{\prime}\right)$. Since $\Omega_{\varrho}$ is cyclic, there exists $Q_{1} \in \mathfrak{A}$ such that $\left(Q_{1} \Omega_{\varrho},\left(E_{b^{\prime}-0}-E_{a^{\prime}+0}\right) \Phi\right) \neq 0$.

Let

$$
\mathrm{d} \mu(\lambda)=\mathrm{d}\left(Q_{1} \Omega_{\varrho}, E_{\lambda} \Phi\right) .
$$

It is a finite complex measure and its restriction to $J$ is not identically 0 . The set $C_{0}(J)$ of all continuous functions vanishing outside of $J$ is separating for finite measures on $J$. Since $C^{\infty}$ functions vanishing outside of $J$ is norm dense in $C_{0}(J)$, there exists a $C^{\infty}$ function $\tilde{f}(\lambda)$ whose support is in $\bar{J}$ and $\int \tilde{f}(\lambda) \mathrm{d} \mu(\lambda) \neq 0$.

Let $f(t)=(2 \pi)^{-1} \int \tilde{f}(\lambda)^{*} e^{-i t \lambda} \mathrm{d} \lambda . f$ is in $\mathscr{S}$. Define

$$
Q=\int_{-\infty}^{\infty} \bar{\tau}_{\varrho}(t) Q_{1} f(t) d t \in \mathfrak{A} .
$$


Then

$$
\begin{aligned}
Q \Omega_{\varrho} & =\int \tilde{f}(\lambda)^{*} \mathrm{~d} E_{\lambda} Q_{1} \Omega_{\varrho} ; \\
\left(Q \Omega_{\varrho}, \Phi\right) & =\int \tilde{f}(\lambda) \mathrm{d} \mu(\lambda) \neq 0 .
\end{aligned}
$$

Lemma 5 is proved if we show $Q \in \mathfrak{A}_{J}$. This follows from the next Lemma. Q.E.D.

Lemma 6. Let $\tilde{f}(\lambda)$ be a $C^{\infty}$ function with its support in a compact interval $\bar{J}$ and

$$
f(t)=(2 \pi)^{-1} \int \tilde{f}(\lambda) e^{-i t \lambda} \mathrm{d} \lambda .
$$

Then, for any $Q_{1}$ in a $\bar{\tau}_{\varrho}$-invariant norm closed linear set $\mathfrak{A}$,

$$
Q(f) \equiv \int_{-\infty}^{\infty} \bar{\tau}_{\varrho}(t) Q_{1} f(t) \mathrm{d} t \in \mathfrak{U}_{J}
$$

Proof. Let $I$ be a bounded open interval and $I_{1}$ be another open interval such that $I_{1} \subset C I$. Since the union of $H\left(I_{1}\right)$ for all such $I_{1}$ is dense in $H(I)$, it is enough to prove that for $\Phi \in H\left(I_{1}\right)$ and any $\Psi$ such that the measure $\mathrm{d}\left(\Psi, E_{\lambda} \Psi\right)$ has a compact support with empty intersection with $I+\bar{J}(=I+J), Q(f)$ satisfies

Let

$$
(\Psi, Q(f) \Phi)=0
$$

$$
F(t, s)=\left(\Psi, \Delta_{\varrho}^{i t} Q_{1} \Delta_{\varrho}^{-i s} \Phi\right) .
$$

$F$ is a uniformly bounded continuous function of $(t, s)$, analytic in $t$ and $s$. Its Fourier transform

$$
\tilde{F}(p, q)=\int e^{i(-p t+q s)} F(t, s) \mathrm{d} t \mathrm{~d} s /(2 \pi)^{2}
$$

is a tempered distribution with support in the direct product of the support of $\mathrm{d}\left(\Psi, E_{\lambda} \Psi\right)$ and $\bar{I}_{1} \subset I$. This support has an empty intersection with the support of $\tilde{f}(p-q)$, which is a $C^{\infty}$ function. Hence

$$
\begin{aligned}
0 & =\int \tilde{F}(p, q) \tilde{f}(p-q) \mathrm{d} p \mathrm{~d} q \\
& =\int F(t, s) f(t) \delta(t-s) \mathrm{d} t \mathrm{~d} s \\
& =\int F(t, t) f(t) \mathrm{d} t=(\Psi, Q(f) \Phi) . \quad \text { Q.E.D. }
\end{aligned}
$$

Lemma 7. $\mathfrak{U}_{J}^{*} \subset \hat{\mathfrak{R}}_{-J}$.

Proof. Let $I_{1}$ and $I_{2}$ be open bounded intervals such that $I_{1}+J$ and $I_{2}$ has an empty intersection. Then $\mathfrak{A}_{J} H\left(I_{1}\right) \perp H\left(I_{2}\right)$. Hence $\mathfrak{A}_{J}^{*} H\left(I_{2}\right)$ $\perp H\left(I_{1}\right)$. Given an open bounded interval $I$. Let $I_{2} \subset C I$. Then $I_{2}-J \subset C I-J$ and $\mathfrak{U}_{J}^{*} H\left(I_{2}\right) \perp H\left(I_{1}\right)$ whenever $I_{2}-J$ has an empty intersection with an open bounded interval $I_{1}$. Since $I_{2}-J C C I-J$, this implies $\mathfrak{A}_{J}^{*} H\left(I_{2}\right)$ $C H(I-J)$. Since the union of $H\left(I_{2}\right)$ is dense in $H(I)$, we have $\mathfrak{A}_{J}^{*} H(I)$ $\subset H(I-J)$ and hence $\mathfrak{U}_{J}^{*} \subset \hat{\mathfrak{R}}_{-J}$. Q.E.D. 
Proof of Theorem 2 when $n=1$. Assume that

$$
\operatorname{dim} H((-\delta, \delta))=1
$$

for some $\delta>0$. Since the spectrum of $\log \Delta_{\varrho}$ is symmetric due to $J_{\varrho}\left(\log \Delta_{\varrho}\right) J_{\varrho}=-\log \Delta_{\varrho}$, there exists $t \geqq \delta$ in the spectrum of $\Delta_{\varrho}$ if $\operatorname{dim} H_{Q}>1$. By Lemma 5, there exist $Q \in \hat{\mathfrak{R}}_{I}, I \subset C(t-\delta / 4, t+\delta / 4)$, such that $\left\|Q \Omega_{e}\right\|=1$, because $H((t-\delta / 4, t+\delta / 4)) \neq 0$. Let

$$
\Phi=Q \Omega_{e} \in H((t-\delta / 4, t+\delta / 4)) .
$$

By Lemma 7 and (2.1), we have $Q^{*} Q \in \hat{\mathfrak{R}}((-\delta / 2, \delta / 2))$ and hence

$$
Q^{*} Q \Omega_{\varrho}=c \Omega_{\varrho}
$$

for some complex number $c$, which is determined by

$$
c=\left\|Q \Omega_{\varrho}\right\|^{2}=1 .
$$

Since $\Omega_{\varrho}$ is separating for $\Re$, we have $Q^{*} Q=1$. Hence $\left\|Q^{*}\right\|=1$.

We now have

$$
\begin{aligned}
1 & \geqq\left\|Q^{*} \Omega_{\varrho}\right\|=\left\|J_{\varrho} Q^{*} \Omega_{\varrho}\right\|=\left\|\Delta_{\varrho}^{1 / 2} Q \Omega_{\varrho}\right\| \\
& =\left\|\Delta_{\varrho}^{1 / 2} \Phi\right\| \geqq\{\exp (1 / 2)(t-\delta / 4)\}\|\Phi\| \\
& >1,
\end{aligned}
$$

which is a contradiction. Q.E.D.

Proof of Theorem 2 for a general $n$. Let $H_{0}$ be the set of all $\Delta_{e}$ invariant vectors in $H_{\varrho}$ and $\hat{\mathfrak{R}}_{0}$ be the set of all $\bar{\tau}_{\varrho}(t)$ invariant elements of $\hat{\mathfrak{R}}=\pi_{\varrho}(\mathfrak{R})$. By assumption, there exists $\delta>0$ such that $H(I)=H_{0}$ for $I=(-\delta, \delta) \cdot \operatorname{dim} H_{0}=n$.

For any $J \subset C I, Q \in \hat{\mathfrak{R}}_{J}$ satisfies $Q \Omega_{\varrho} \in H(I)=H_{0}$ because $\Omega_{\varrho} \in H\left(I_{1}\right)$ for small $I_{1}$ containing 0 such that $J+I_{1} \subset I$. Hence $\left\{\bar{\tau}_{e}(t) Q\right\} \Omega_{Q}=\Delta_{e}^{i t} Q \Omega_{Q}$ $=Q \Omega_{\varrho}$. Since $\Omega_{\varrho}$ is separating, $\bar{\tau}_{\varrho}(t) Q=Q$ and hence $\mathfrak{R}(I) \subset \hat{\mathfrak{R}}_{0}$. If $0 \in J$, then $\mathfrak{R}(J) \supset \hat{\mathfrak{R}}_{0}$. Hence $\hat{\mathfrak{R}}(J)=\hat{\mathfrak{R}}_{0}$ for $J \subset I$. By Lemma $5, \hat{\mathfrak{R}}_{0} \Omega_{\varrho}$ is dense in $H(I)=H_{0}$ and hence $\hat{\mathfrak{R}}_{0} \Omega_{\varrho}=H_{0}$. Since $\Omega_{\varrho}$ is separating for $\hat{\mathfrak{R}}$, it is cyclic and separating for $\hat{\mathfrak{R}}_{0}$ in $H_{0}$. By KMS condition, $\Omega_{\varrho}$ is a trace vector for $\hat{\mathfrak{R}}_{0}$.

There exists a set of mutually orthogonal minimal projections $s_{i} \in \hat{\mathfrak{R}}_{0}$ such that $\Sigma s_{i}=1$. Let $\Omega_{i}=s_{i} \Omega_{\varrho}$. Since $J_{\varrho} s_{i} \Omega_{\varrho}=s_{i} \Omega_{\varrho}$ because $\Delta_{\varrho}$ is 1 on $H_{0}$, we have $s_{i} \Omega_{Q}=j_{\varrho}\left(s_{i}\right) \Omega_{\varrho}=s_{i}{ }^{2} \Omega_{\varrho}=s_{i} j_{\varrho}\left(s_{i}\right) \Omega_{\varrho}$. Let $s_{i} j_{\varrho}\left(s_{i}\right) H=H_{i}$. Then $\Omega_{i}=s_{i} \Omega_{\varrho} \in H_{i}$. Since $\left(s_{i} \mathfrak{R} s_{i} \Omega_{i}\right)^{-}=\left(s_{i} \mathfrak{R} j\left(s_{i}\right) \Omega_{\varrho}\right)^{-}=\left(s_{i} j\left(s_{i}\right) \mathfrak{R} \Omega_{\varrho}\right)^{-}=H_{i}, \Omega_{i}$ is cyclic for $\mathfrak{R}_{i} \equiv s_{i} \hat{\mathfrak{R}} s_{i}$. Since $Q \Omega_{i}=Q \Omega_{\varrho}$ for $Q \in \mathfrak{R}_{i}, \Omega_{i}$ is separating for $\mathfrak{R}_{i}$. For $Q \in \mathfrak{R}_{i}$, we have

$$
\begin{aligned}
S_{\varrho} Q \Omega_{i} & =S_{\varrho} Q s_{i} \Omega_{\varrho}=S_{\varrho} Q \Omega_{\varrho}=Q^{*} \Omega_{\varrho} \\
& =Q^{*} \Omega_{i},
\end{aligned}
$$

where $S_{\varrho}=J_{\varrho} \Delta_{\varrho}^{1 / 2}$. Hence the restriction of $J_{\varrho}$ and $\Delta_{\varrho}$ are $J_{\Omega_{i}}$ and $\Delta_{\Omega_{i}}$ in $H_{i}$. 
Since $s_{i}$ is minimal in $\hat{\mathfrak{R}}_{0}$ and $\Omega_{\varrho}$ is cyclic separating trace vector, $j_{\varrho}\left(s_{i}\right)$ is minimal in the commutant of $\hat{\mathfrak{R}}_{0}$ in $H_{0}$ and $\Omega_{i}=s_{i} j_{\varrho}\left(s_{i}\right) \Omega_{\varrho}$ spans $s_{i} j_{\varrho}\left(s_{i}\right) H_{0}$. Hence $\Delta_{\Omega_{i}}$ has an isolated spectrum at 1 with multiplicity 1 and hence $\operatorname{dim} H_{i}=1$. Hence $s_{i} \mathfrak{R} s_{i}=\mathfrak{R}_{i} \sim C$. Therefore $s_{i}$ is also a minimal projection of $\mathfrak{R}$. Since the number of $s_{i}$ can not exceed $\operatorname{dim} H_{0}=n$, $\Re$ has at most $n$ mutually orthogonal minimal projections with sum 1 . This implies $\operatorname{dim} H_{\varrho} \leqq n^{2}$. Q.E.D.

\section{§ 4. Isolated Spectrum with a Finite Multiplicity at $x \neq 1$}

Theorem 3. If $x$ is an isolated spectrum of $\Delta_{\varrho}$ with a finite multiplicity, then there exists a direct sum decomposition

$$
\pi_{\varrho}(\Re)=\Re_{a} \oplus \Re_{b}, \quad \Omega_{\varrho}=\Omega_{a} \oplus \Omega_{b}, \quad \Delta_{\varrho}=\Delta_{\Omega_{a}} \oplus \Delta_{\Omega_{b}}
$$

such that $\mathfrak{R}_{a}$ is of type I with a finite atomic center and $\Delta_{\Omega_{b}}$ does not have its spectrum at $x$ and $x^{-1}$.

Let $H_{t}$ denote the set of all eigenvectors of $\Delta_{\varrho}$ belonging to an eigenvalue $e^{t}$ and $s_{t}$ be the projection to the subspace spanned by $\hat{\mathfrak{R}}^{\prime} H_{t}$ $+\hat{\mathfrak{R}}^{\prime} H_{-t}$. As a preparation for our proof, we have the following:

Lemma 8. Assume that $H((t-\delta, t+\delta))=H_{t}$.

Then

(a) $\left[s_{t}, \Delta_{\varrho}\right]=0$.

(b) 1 is an isolated spectrum of $\Delta_{\varrho} \mid s_{t} H$.

(c) If $\operatorname{dim} H_{t}<\infty$, then $\operatorname{dim} s_{t} H_{0}<\infty$.

(d) $\left(1-s_{t}\right) \Delta_{\varrho}$ does not have its spectrum at $e^{ \pm t}$.

Proof. If $t=0$, then $s_{t}=1$ and all statements become trivial. Hence we assume $t \neq 0$.

(a) Since $H_{t}$ and $H_{-t}$ are invariant under $\Delta_{\varrho}^{i t}$ and $\hat{\mathfrak{R}}^{\prime}$ is invariant under $\bar{\tau}_{\varrho}(t), s_{t} H_{\varrho}$ is invariant under $\Delta_{\varrho}^{i t}$ and hence $\left[s_{t}, \Delta_{\varrho}\right]=0$.

(b) For any $J \subset \subset(-\delta, \delta)$, there exists $I \ni t$ such that $J+I \subset(t-\delta, t+\delta)$. Then $H_{t} \subset H(I)$ and $\hat{\mathfrak{R}}_{J} H_{t} \subset H((t-\delta, t+\delta))=H_{t}$. For $Q \in \hat{\mathfrak{R}}_{J}$ and $\Psi \in H_{t}$,

Hence

$$
\bar{\tau}_{\varrho}(u) Q \Psi=e^{-i u} \Delta_{\varrho}^{i u} Q \Psi=Q \Psi .
$$

for all $\Psi \in \hat{\mathfrak{R}}^{\prime} H_{t}$.

$$
\left\{\bar{\tau}_{\varrho}(u) Q-Q\right\} \Psi=0
$$

Since $J_{\varrho} \Delta_{\varrho} J_{\varrho}=\Delta_{\varrho}^{-1}, \log \Delta_{\varrho}$ has a symmetric spectrum and hence $H((-t-\delta,-t+\delta))=H_{-t}$. By the same argument as above, (4.1) holds for $\Psi \in \hat{\mathfrak{R}}^{\prime} H_{-t}$ and hence for $\Psi \in s_{t} H_{\varrho}$. We have

$$
\bar{\tau}_{\varrho}(u)\left\{Q s_{t}\right\}=\left\{\bar{\tau}_{\varrho}(u) Q\right\} s_{t}=Q s_{t} .
$$

Hence $\hat{\mathfrak{R}}_{J} s_{t} \subset \hat{\mathfrak{R}}_{0}$ for any. $J \subset C(-\delta, \delta)$. Clearly, $\hat{\mathfrak{R}}_{0} s_{t} \subset \hat{\mathfrak{R}}_{J} s_{t}$. Hence $\hat{\mathfrak{R}}_{J} s_{t}$ $=\hat{\mathfrak{R}}_{0} s_{t}$. Taking adjoint, $\mathrm{s}_{t} \hat{\mathfrak{R}}_{J}=s_{t} \hat{\mathfrak{R}}_{0}$. 
By Lemma $5, s_{t} H((-\delta, \delta))$ is generated by

$$
s_{t} \hat{\mathfrak{R}}_{J} \Omega_{\varrho}=s_{t} \hat{\mathfrak{R}}_{0} \Omega_{\varrho} \subset s_{t} H_{0}, \quad J \subset C(-\delta, \delta) .
$$

Hence 1 is an isolated spectrum of $\left.\Delta_{\varrho}\right|_{s_{t} H}$. Moreover, $s_{t} H_{0} \subset s_{t} \hat{\mathfrak{R}}_{J} \Omega_{\varrho}$ $C s_{t} H_{0}$ and hence $s_{t} \hat{\mathfrak{R}}_{0} \Omega_{\varrho}=s_{t} H_{0}$.

(c) $\operatorname{dim} H_{t}<\infty$ implies $\operatorname{dim} H_{-t}=\operatorname{dim} J_{\varrho} H_{t}<\infty$. Since $Q H_{t}=0$, $Q H_{-t}=0, Q \in \hat{\mathfrak{R}}$ imply $Q s_{t}=0$, we have

$$
\operatorname{dim} H_{t}+\operatorname{dim} H_{-t} \geqq \operatorname{dim} \hat{\mathfrak{R}}_{J} s_{t}=\operatorname{dim} \hat{\mathfrak{R}}_{0} s_{t}=\operatorname{dim} s_{t} H_{0} .
$$

(d) This follows from (1) and the definition of $s_{t}$. Q.E.D.

Proof of Theorem 3. Let $x=e^{t}$. If $t=0$, then Theorem 3 holds with $\mathfrak{R}_{b}=0$ due to Theorem 2. Assume that $t \neq 0$. Let

$$
\begin{aligned}
K & =s_{t} j_{\varrho}\left(s_{t}\right) H_{\varrho}, \\
\mathfrak{M} & =\left.s_{t} \hat{\mathfrak{R}} s_{t}\right|_{K}, \\
\Psi & =s_{t} j_{\varrho}\left(s_{t}\right) \Omega_{\varrho} .
\end{aligned}
$$

By (a) of Lemma 8, we have $s_{t} \Omega_{\varrho}=\Delta_{\varrho}^{1 / 2} s_{t} \Omega_{\varrho}=J_{\varrho} s_{t} \Omega_{\varrho}=j_{\varrho}\left(s_{t}\right) \Omega_{\varrho}$ $=s_{t}{ }^{2} \Omega_{\varrho}=s_{t} j_{\varrho}\left(s_{t}\right) \Omega_{\varrho}=\Psi$. Hence $\mathfrak{M} \Psi=s_{t} \hat{\mathfrak{R}} j_{\varrho}\left(s_{t}\right) \Omega_{e}=s_{t} j_{\varrho}\left(s_{t}\right) \hat{R} \Omega_{\varrho}$ is dense in $K$ and $\mathfrak{M}^{\prime} \Psi^{\varrho}=j_{\varrho}\left(s_{t}\right) \hat{\mathfrak{R}}^{\prime} j_{\varrho}\left(s_{t}\right) s_{t} \Omega_{\varrho}=j_{\varrho}\left(s_{t}\right) \hat{\mathfrak{R}}^{\prime} s_{t} \Omega_{\varrho}=j_{\varrho}\left(s_{t}\right) s_{t} \hat{\mathfrak{R}}^{\prime} \Omega_{\varrho}$ is also dense in $K$. Hence $\Psi$ is cyclic and separating for $\mathfrak{M}$ in $K$. For $Q \in \mathfrak{M}$ and $S_{\varrho}=J_{\varrho} \Delta_{\varrho}^{1 / 2}$,

$$
S_{\varrho} Q s_{t} \Omega_{\varrho}=s_{t} Q^{*} \Omega_{\varrho}=Q^{*} s_{t} \Omega_{\varrho}
$$

and hence $\left.S_{\varrho}\right|_{K}=S_{\Psi},\left.\Delta_{\varrho}\right|_{K}=\Delta_{\Psi}$ and $\left.J_{\varrho}\right|_{K}=J_{\Psi}$.

By (c) of Lemma $8, \Delta_{\Psi}$ has an isolated spectrum with a finite multiplicity. Hence $\mathfrak{M}$ is a finite matrix algebra by Theorem 2 .

Since $s_{t} H_{t}=H_{t}, j_{\varrho}\left(s_{t}\right) H_{t}=J_{\varrho} s_{t} J_{\varrho} H_{t}=J_{\varrho} s_{t} H_{-t}=J_{\varrho} H_{-t}=H_{t}$. Similarly $j_{\varrho}\left(s_{t}\right) H_{-t}=H_{-t}$. Hence $H_{t}+H_{-t} \subset K$.

Let $c\left(s_{t}\right)$ be the central support of $s_{t}$. Since $j_{\varrho}\left(c\left(s_{t}\right)\right)=c\left(s_{t}\right)$ (for any central projection), $c\left(j_{\varrho}\left(s_{t}\right)\right)=c\left(s_{t}\right) . \mathfrak{M}$ is isomorphic to $s_{t} \mathfrak{R} s_{t}$ restricted to $\hat{\mathfrak{R}}^{\prime} K=s_{t} \hat{\mathfrak{R}}^{\prime} \Omega_{\varrho}=s_{t} H$. Hence $c\left(s_{t}\right) \hat{\mathfrak{R}}$ must be of type $I$ with a finite atomic center.

$$
\mathfrak{R}_{a}=c\left(s_{t}\right) \hat{\mathfrak{R}}, \quad \mathfrak{R}_{b}=\left(1-c\left(s_{t}\right)\right) \hat{\mathfrak{R}}, \Omega_{a}=c\left(s_{t}\right) \Omega_{\varrho},
$$

$\Omega_{b}=\left(1-c\left(s_{t}\right)\right) \Omega_{\varrho}$ satisfy required properties. Q.E.D.

\section{§5. Applications}

Connes has introduced the invariant

$$
S(\Re)=\bigcap_{\varrho} \operatorname{spectrum} \Delta_{\varrho} .
$$

Our result gives the following application for $S(\mathfrak{R})$. 
Theorem 4. Let $\varrho$ be a faithful normal state of $\mathfrak{R}$ invariant under a net of * automorphisms $\tau_{\alpha}$ of $\mathfrak{R}$. Assume that $\mathfrak{R}$ has a weakly dense sub * algebra $\mathfrak{U}$ which is strongly $\tau_{\alpha}$ central. Then

$$
S(\Re)=\operatorname{Spectrum} \Delta_{\varrho} .
$$

If $\varrho$ is ergodic with respect to modular automorphisms in addition, then either $S(\mathfrak{R})$ is $[0, \infty)$ or $H_{\varrho}$ is of one dimension.

Proof. The first half follows from Theorem 1. If $\varrho$ is $\tau_{\varrho}$ ergodic, then $\varrho$ is primary and hence Spectrum $\Delta_{\varrho} \backslash\{0\}$ is a multiplicative group. If 1 is not an isolated spectrum of $\Delta_{\varrho}$, then Spectrum $\Delta_{\varrho}=[0, \infty)$. If 1 is an isolated spectrum of $\Delta_{\varrho}$, then Theorem 2 is applicable where $n=1$ due to $\tau_{\varrho}$ ergodicity. Hence $\operatorname{dim} H_{\varrho}=1$. Q.E.D.

Remark 1. Størmer [4] proved the first part under the assumption of strong clustering. The second part is stated in [4] with the assumption that $\tau_{\varrho}$ is asymptotically abelian.

Theorem 5. $S(\mathfrak{R})=\bigcap_{\varrho}$ essential spectrum $\Delta_{\varrho}$.

Proof. Obvious from Theorem 3. Q.E.D.

Remark 2. Connes invariant is additive under direct sum $S\left(\mathfrak{R}_{1} \oplus \mathfrak{R}_{2}\right)$ $=S\left(\mathfrak{R}_{1}\right) \cup S\left(\mathfrak{R}_{2}\right)$, whereas the asymptotic ratio set satisfies $r_{\infty}\left(\mathfrak{R}_{1} \oplus \mathfrak{R}_{2}\right)$ $=r_{\infty}\left(\mathfrak{R}_{1}\right) \cap r_{\infty}\left(\mathfrak{R}_{2}\right) . S(\mathfrak{R})$ is more closely related to the union of $S_{x}$ over non-zero portion of partial central decomposition of $\mathfrak{R}$ according to asymptotic ratio set.

Remark 3. In the situation of Theorem 4, if $\mathfrak{R}$ is ITPFI, then $\mathfrak{R}=\mathfrak{R}_{x}$, $0<x \leqq \infty$. If $\varrho$ is $\tau_{\varrho}$ ergodic, then $\mathfrak{R}=\mathfrak{R}_{\infty} . \mathfrak{R}$ appearing in Gibbs states of a lattice system is hyperfinite but it is not known whether it is an ITPFI in general.

\section{Appendix}

The following result is a part of Theorem 4 in [1] and is a basis for Lemma 1 of $\S 1$.

Lemma 9. If $Q_{\alpha}$ is a uniformly bounded weakly central net in $R$ and if $\varrho$ and $\varrho^{\prime}$ are normal states of $\mathfrak{R}$ such that $\varrho(z)=\varrho^{\prime}(z)$ for all $z \in \mathcal{Z}=\left(\mathfrak{R} \cap \mathfrak{R}^{\prime}\right)$, then

$$
\lim \left\{\varrho\left(Q_{\alpha}\right)-\varrho^{\prime}\left(Q_{\alpha}\right)\right\}=0 .
$$

The following direct proof is due to Elliott.

Proof. Let $Q_{\alpha(\beta)}$ be weakly converging subnet of $\mathrm{Q}_{\alpha}$. Since $Q_{\alpha}$ is weakly central,

$$
z=\mathrm{w}-\lim Q_{\alpha(\beta)} \in 3 \text {. }
$$


Hence $\varrho(z)=\varrho^{\prime}(z)$, i.e.

$$
\lim \left\{\varrho\left(Q_{\alpha(\beta)}\right)-\varrho^{\prime}\left(Q_{\alpha(\beta)}\right)\right\}=0 .
$$

In view of weak compactness of the unit ball of $\mathfrak{R}$, this implies (A.1).

Q.E.D.

Somewhat stronger conclusion can be drawn if $Q_{\alpha}=\tau_{\alpha} Q$, and $\varrho$ is a faithful invariant state. An example is seen in the following:

Lemma 10. Let $\mathfrak{A}$ be a weakly dense * subalgebra of $\mathfrak{R}, \varrho$ be a faithful normal state of $\mathfrak{R}, \tau_{\alpha}$ be a net of * automorphisms of $\mathfrak{R}$ such that $\varrho$ is invariant and $\mathfrak{A}$ is weakly $\tau_{\alpha}$ central, and $\varrho^{\prime}$ be a normal state of $\mathfrak{R}$ such that $\varrho^{\prime}(z)=\varrho(z)$ for every $z \in \Re \cap \Re^{\prime}$. Then

$$
\lim \varrho^{\prime}\left(\tau_{\alpha} Q\right)=\varrho(Q), \quad Q \in \mathfrak{R} .
$$

Proof. By Theorem 4 of [1],

$$
\mathrm{w}-\lim \left\{\tau_{\alpha} Q_{1}-\tau_{\alpha} F_{Q}^{3 \Re}\left(Q_{1}\right)\right\}=0
$$

for $Q_{1} \in \mathfrak{A}$, which implies

$$
\mathrm{w}-\lim U_{\alpha} \pi_{\varrho}\left(Q_{1}-F_{\varrho}^{3 \Re}\left(Q_{1}\right)\right) \Omega_{\varrho}=0 .
$$

Since $F_{Q}^{3 \mathfrak{R}}$ is strongly continuous on the unit ball, there exists $Q_{1} \in \mathfrak{A}$ for given $Q \in \Re, \Phi_{j} \in H_{e}, j=1 \ldots n$, and $\varepsilon>0$ such that

$$
\left\|\left\{\pi_{\varrho}\left(Q_{1}-F_{\varrho}^{3 \Re}\left(Q_{1}\right)\right)-\pi_{\varrho}\left(Q-F_{\varrho}^{3 \Re}(Q)\right)\right\} \Omega_{\varrho}\right\|\left\|\Phi_{j}\right\|<\varepsilon / 2 .
$$

For this $Q_{1}$, there exists $\alpha_{0}$ such that for $\alpha>\alpha_{0}$,

$$
\left|\left(\Phi_{j}, U_{\alpha} \pi_{\varrho}\left(Q_{1}-F_{\varrho}^{3 \Re}\left(Q_{1}\right)\right) \Omega_{\varrho}\right)\right|<\varepsilon / 2 .
$$

These two equations imply

and hence

$$
\left|\left(\Phi_{j}, U_{\alpha} \pi_{\varrho}\left(Q-F_{\varrho}^{3 \Re}(Q)\right) \Omega_{\varrho}\right)\right|<\varepsilon
$$

$$
\mathrm{w}-\lim \pi_{\varrho}\left(\tau_{\alpha}\left\{Q-F_{\varrho}^{3 \Re}(Q)\right\}\right) \Omega_{\varrho}=0 .
$$

Multiplying $Q^{\prime} \in \pi_{\varrho}(\Re)^{\prime}$ and using the cyclicity of $\Omega_{\varrho}$ for $\pi_{\varrho}(\Re)^{\prime}$, we obtain

which implies

$$
\mathrm{w}-\lim \pi_{\varrho}\left(\tau_{\alpha} Q-\tau_{\alpha} F_{\varrho}^{3 \Re}(Q)\right)=0,
$$

$$
\mathrm{w}-\lim \left(\tau_{\alpha} Q-\tau_{\alpha} F_{\varrho}^{3 \Re}(Q)\right)=0, \quad Q \in \Re .
$$

Since $F_{\varrho}^{3 \Re}(Q) \in \mathcal{3}$, we obtain

$$
\varrho^{\prime}\left(\tau_{\alpha} F_{\varrho}^{3 \Re}(Q)\right)=\varrho\left(\tau_{\alpha} F_{\varrho}^{3 \Re}(Q)\right)=\varrho\left(F_{\varrho}^{3 \Re}(Q)\right)=\varrho(Q) .
$$


Hence we obtain from (A.3)

$$
\begin{array}{rlr}
\lim \varrho^{\prime}\left(\tau_{\alpha} Q\right) & =\lim \varrho^{\prime}\left(\tau_{\alpha} F_{\varrho}^{3 \Re}(Q)\right) \\
& =\varrho(Q) . &
\end{array}
$$

Acknowledgement. The author would like to thank Professor E. J. Woods and Dr. G. Elliott for helpful discussions and warm hospitality at Department of Mathematics, Queen's University.

\section{References}

1. Araki, H.: Normal positive linear mappings of norm 1 from a von Neumann Algebra into its commutant and its application. (Queen's Mathematical preprint 1972-12.)

2. Connes, A.: C.R. Acad. Sci. Paris 273, 900-903 (1971).

3. Connes, A.: C.R. Acad. Sci. Paris 274, 175-177 (1972).

4. Størmer,E.: Spectra of states and asymptotically abelian $C^{*}$ algebras. (Oslo Mathematics preprint No. 8,1972 .)

H. Araki

Research Institute for Mathematical Sciences Kyoto University

Kyoto, Japan 
